\title{
Shear moduli of two dimensional binary glasses
}

\author{
R Seyboldt, ${ }^{a}$ D Hajnal, ${ }^{b}$ F Weysser ${ }^{a}$ and M Fuchs ${ }^{* a}$
}

\section{DOI: $10.1039 / \mathrm{c} 2 \mathrm{sm} 07010 \mathrm{c}$}

The shear moduli of two-component glasses in two dimensions are studied within mode coupling theory. Varying the concentration, strong mixing effects are observed along the glass transition lines for two interaction potentials. Nonoverlapping disks with size ratios between 0.3 and 0.9 , and point particles interacting with (magnetic) dipoles of strength ratio between 0.1 and 0.6 are considered. Equilibrium structure factors (partially obtained from Monte Carlo simulations) and glass form factors, and perturbative calculations show that a softening of the elastic shear constant of glass upon adding another component arises from a dilution effect of the majority component. For very disparate mixtures, an anomalous elastic strenghtening results from what we interpret as clustering of the smaller particles in the voids between the larger ones. This might point to a glass-glass transition. We include simulation data on hard disk mixtures which show that the theory underestimates the moduli by around $50 \%$, but otherwise captures the qualitative trends (within the rather large simulational error bars).

\section{Introduction}

Mixtures often are good glass formers as mixing suppresses the tendency of the liquid to crystallize. It is also well known and often desired that mixing can have strong effects on the viscosity and diffusivities of the supercooled liquid. ${ }^{1,2}$ For a recent study of a colloid-polymer mixture where the Newtonian viscosity could rationally be changed by more than two orders see ref. 3 Equally important are mixing effects in glass itself, e.g. in glasses formed from multicomponent metallic alloys. ${ }^{4,5}$ An especially rich scenario in mixtures including polyamorphism, viz. the existence of different amorphous states with possible glass-to-glass transitions in between, has been discussed for metallic glass and could open a way to tune its elastic properties. ${ }^{6}$ Glass-to-glass transitions are also expected in the quoted soft matter system of a colloid-polymer mixture and there linked to the viscosity variation. ${ }^{3}$ Despite the interest, few studies within atomistic theory exist which address the microscopic origins of mixing effects in glass. Mode coupling theory (MCT), which gives a microscopic description of the viscoelasticity in weakly supercooled melts and of dispersions at the colloidal glass transition, has been extended to multicomponent mixtures. ${ }^{7}$ Results for the shear elasticity of binary mixtures of hard spheres exist, ${ }^{8}$ and comparisons of structural and transport properties with neutron scattering experiments on metallic alloys proved promising., ${ }^{9,10}$ In computer simulations the MCT approximations entering the shear modulus formula were directly tested with again promising findings. ${ }^{11}$

"Fachbereich Physik, Universität Konstanz, 78457 Konstanz, Germany ${ }^{b}$ Institut für Physik, Johannes Gutenberg-Universität Mainz, 55099 Mainz, Germany
In this paper, the shear modulus, viz. the transverse elastic constant, of binary glasses of hard disks and of dipolar particles is presented. Because of recent advances to prepare and study by microscopy two-dimensional glasses using binary colloidal dispersions, ${ }^{12}$ we specialize our numerical investigations to $d=2$ spatial dimensions. MCT gives two different formulae of the shear modulus $G_{\infty}$, where the connection to the mean-squared displacement fluctuations is the one found very useful in the colloid experiments. ${ }^{13}$ The other relation identifies $G_{\infty}$ as the frozen-in (nonergodic) contribution of the shear-stress autocorrelation function. Importantly, MCT predicts $G_{\infty}$ uniquely from the equilibrium static structure factor $S_{q}$ so that related formulae hold for macromolecular dispersions and molecular and atomic alloys. We thus can give the shear modulus as function of the thermodynamic state point in the following without having to discuss kinetic effects. In the notation $G_{\infty}$ we follow Maxwell, who identified the value of the shear modulus at the glass transition, to be denoted as $G_{\infty}^{c}$, as the amplitude of the so-called $\alpha$-process (with relaxation time $\tau_{\alpha}$ ) in the supercooled fluid; it is accessible at high frequencies $\omega \tau_{\alpha} \gg 1$, which motivates the index.

The paper is organized as follows: section 2 defines the studied mixtures and recalls the pertinent formulae from the microscopic theory, while Sect. 3 gives numerical implementation details. In Sect. 4 the results are discussed and compared to computer simulation data. A short summary concludes our manuscript.

\section{Theoretical background}

\subsection{The shear modulus in mode coupling theory}

The derivation of a formula in MCT to calculate the shear modulus, viz. the shear stress auto-correlation function 
$G(t)=\frac{1}{V}\left\langle\sigma_{x y}(t) \sigma_{x y}\right\rangle$, from the matrix of partial structure factors $S(k)$ and the matrix of partial intermediate scattering functions $S(k, t)$ in $d$ dimensions is done analogously to the derivation in three dimensions. A careful exposition aimed at colloidal mixtures has been given by Nägele and Bergenholtz. ${ }^{14}$ Only trivial adaptations of their derivation are necessary since the more abstract parts are independent of dimension. One gets for the $d$-dimensional system (with $d>1$ )

$$
G(t)=\frac{k_{B} T}{2} \frac{1}{(2 \pi)^{d}} \int_{0}^{\infty} \phi\left(\frac{k_{x} k_{y}}{k}\right)^{2} d \Omega_{k} \operatorname{Tr}\left[\left(\frac{d C(k)}{d k} S(k, t)\right)^{2}\right] d k .
$$

For $d=2$ the integral over the unity-sphere in k-space $\phi d \Omega_{k}$ has to be evaluated. This yields

$$
G(t)=\frac{k_{B} T}{32 \pi} \int_{0}^{\infty} k^{3} \operatorname{Tr}\left[\left(\frac{d C(k)}{d k} S(k, t)\right)^{2}\right] d k .
$$

The matrix of partial intermediate scattering functions $S_{\alpha \beta}(k, t)=\left(N_{\alpha} N_{\beta}\right)^{-\frac{1}{2}}\left\langle\left[\delta \rho_{\alpha}(\vec{k}, t)\right] * \delta \rho_{\beta}(\vec{k}, 0)\right\rangle$ describes the timeand wave vector dependence of density fluctuations $\delta \rho_{\alpha}(\vec{k}, t)=\sum_{m=1}^{N_{\alpha}} \exp \left(i \vec{k} \cdot \vec{r}_{\alpha, \mathrm{m}}(t)\right)$ in thermal equilibrium with $\alpha$, $\beta \in s, b$ where index $b$ stands for big, $s$ for small species. It is symmetric and reduces to the matrix of equilibrium partial structure factors at zero time, $S(k, t=0)=S(k)$. Via the Ornstein-Zernike equation, the latter is connected to the matrix of direct correlation functions $C$. We use the convention

$$
\begin{aligned}
C_{b b} & =1-\frac{S_{s s}}{S_{b b} S_{s s}-S_{b s}^{2}}, \\
C_{b s} & =\frac{S_{b s}}{S_{b b} S_{s s}-S_{b s}^{2}}, \\
C_{s s} & =1-\frac{S_{b b}}{S_{b b} S_{s s}-S_{b s}^{2}} .
\end{aligned}
$$

The plateau shear modulus $G_{\infty}:=G(t \rightarrow \infty)$ is obtained, when in an idealized glass density fluctuations do not decorrelate at long times: $\lim _{t \rightarrow \infty} S(k, t)=F(k)$. The symmetric matrix $F$ is termed glass form factors or nonergodicity parameters. ${ }^{7} \mathrm{~A}$ frozen-in or nonergodic contribution in the shear stress autocorrelator results, which is the glass (or plateau) shear modulus

$$
G_{\infty}=\frac{k_{B} T}{32 \pi} \int_{0}^{\infty} k^{3} \operatorname{Tr}\left[\left(\frac{d C(k)}{d k} F(k)\right)^{2}\right] d k .
$$

With $C^{\prime}=\frac{d C}{d k}$, the trace in the above equation becomes

$$
\begin{aligned}
\operatorname{Tr} & =\operatorname{Tr}\left[\left(\frac{d C}{d k} F\right)^{2}\right] \\
& =C_{b b}^{\prime 2} F_{b b}^{2}+2 C_{b b}^{\prime} F_{b s}\left(2 C_{b s}^{\prime} F_{b b}+C_{s s}^{\prime} F_{b s}\right) \\
& +4 C_{b s}^{\prime} C_{s s}^{\prime} F_{b s} F_{s s}+C_{s s}^{\prime 2} F_{s s}^{2}+2 C_{b s}^{\prime 2}\left(F_{b s}^{2}+F_{b b} F_{s s}\right) .
\end{aligned}
$$

We will give the shear modulus as $G_{\infty} /\left(n k_{B} T\right)$, as then the limit of a one-component system will be constant with respect to a variation of the particle size.

\subsection{Asymptotic results close to the glass transition}

MCT predicts that the shear modulus jumps discontinuously from zero in the fluid to a finite value at the glass transition. We will denote the latter value as $G_{\infty}^{c}$. This prediction follows from the bifurcation of the nonergodicity parameters, which vanish in the fluid $S(k, t \rightarrow \infty)=0$, and are finite at and above the transition $S(k, t \rightarrow \infty)=F$. The nonergodicity parameter $F$ can be extrapolated from the value $F_{c}$ at the transition into the glass. With the relative distance from the glass transition $\sigma=c_{1} \frac{\phi-\phi_{c}}{\phi_{c}}$ or $\sigma=c_{2} \frac{\Gamma-\Gamma_{c}}{\Gamma_{c}}$, this is

$$
F(\sigma)=F_{c}+H \sqrt{\frac{\sigma}{1-\lambda}}
$$

Inserting the equation into $G_{\infty}$ yields

$$
G_{\infty}(\sigma)=G_{\infty}^{c}+H_{G} \sqrt{\frac{\sigma}{1-\lambda}}
$$

with

$$
H_{G}=\frac{k_{B} T}{32 \pi} \int_{0}^{\infty} k^{3} \operatorname{Tr}\left[C_{c}^{\prime} H C_{c}^{\prime} F_{c}+C_{c}^{\prime} F_{c} C_{c}^{\prime} H\right] d k
$$

We will use these asymptotic results in order to characterize the magnitude and density/temperature dependence of the shear modulus of two binary mixtures tracking their glass transition lines.

\subsection{Binary Hard disks}

A binary mixture of hard disks is an easy model system possessing a glass transition, where mixing effects can be studied. Here we look at the system in two dimensions, because twodimensional systems can often be more easily visualized experimentally, and because the comparison with the three-dimensional system provides insight into the dependence of the glass transition on the spatial dimension.

The two-dimensional system consists of two kinds of hard disks, with radii $R_{s}$ and $R_{b}$. Here $s$ denotes the smaller and $b$ the bigger particles, so that $R_{s}<R_{b}$. As they are hard disks they cannot overlap, but otherwise they do not interact.

Since we want to study mixing phenomena, we will vary the concentration of the smaller particles and the size ratio $\delta_{R}=R_{s} / R_{b}$. In the following the concentration of small particles is given in terms of the relative concentration of small particles by volume $\hat{x}_{\mathrm{s}}=\phi_{\mathrm{s}} / \phi$, since this is a better mixing parameter than the number density ratio $x_{s}$. It renders the plots overall more symmetric, and at the mixing ratio $\hat{x}_{s}=1 / 2$ both components determine the elasticity close to equally. Note that for disparate sizes, $x_{s}=1 / 2$ describes a very different mixing ratio. For the dimension $d$ the relation between both parameters is

$$
x_{s}=\frac{\hat{x}_{s} / \delta_{R}^{d}}{1+\hat{x}_{s}\left(1 / \delta_{R}^{d}-1\right)} .
$$

To study the system at the glass transition, we have to change the number density $n=N / V$ as well, otherwise the system would become more solid or more liquid. At fixed $x_{s}$ and $\delta$ while 
keeping $R_{b}$ constant, we do this by changing the (total) packing fraction

$$
\phi=n \pi R_{b}^{2}\left(\mathrm{x}_{b}+x_{s} \delta_{R}^{2}\right) .
$$

The system was studied among others by Weysser and Hajnal. ${ }^{15-17}$ It has been found that the two-dimensional MCT results are valid and similar to the results in three dimensions. The three-dimensional system, including the plateau shear modulus, was studied by Götze and Voigtmann. ${ }^{8}$ We will thus be able to compare the results.

\subsection{Binary dipolar point particles}

The second system that we study here is a simplified model of the system studied experimentally by König et al. ${ }^{12}$ They use superparamagnetic colloidal particles at a planar water-air interface and induce a magnetic field so that the colloids repel each other. The particles have mean distances of several radii of their hard cores, so we will ignore the cores. This leads to a simplified model system of dipolar point particles that interact via their magnetic dipole potential

$$
u^{\alpha \beta}(r)=\frac{\mu_{0}}{4 \pi} \frac{\chi_{\alpha} \chi_{\beta} B^{2}}{r^{3}}
$$

The main parameters are $x_{s}$, the (number-) concentration of small particles, and $\delta_{\chi}=\chi_{s} / \chi_{l}$, the ratio of the susceptibilities. The dependence of a thermodynamic state point on total particle number-concentration $n$, temperature $T$, and magnetic field $B$ is given by the coupling parameter ${ }^{12}$

$$
\Gamma=(\pi n)^{3 / 2} \frac{\mu_{0}}{4 \pi} \frac{\left[x_{b}+x_{s} \delta_{\chi}\right]^{2} \chi_{b}^{2} B^{2}}{k_{B} T} .
$$

The fact that the particles are presumed to be point particles leads to the following result. Different from the hard disk system, the number density $n$ does not need to be changed when the mixing parameters are chosen differently: The system can be brought to the glass transition by increasing the external magnetic field thus "pumping up" the particles.

Binary mixtures of this system have been studied theoretically in detail but excluding the elastic moduli. ${ }^{18}$ The model system has been shown to describe the experimental system well in terms of the structure factors. It has further been shown that MCT yields good results for some dynamical quantities. ${ }^{18,19}$

As for the hard disk system, the use of $x_{s}$ puts all interesting features near $x_{s}=1$, especially for small $\delta_{\chi}$. The explanation for this is obvious as $x_{s}$ overestimates the effects of the minoritycomponent when weakly interacting particles are mixed in. Because of this we introduce $\hat{x}_{s}$, comparable to the volumeconcentration of small particles of the hard-disk system. This new $\hat{x}_{s}$ can be defined in two ways. Either by taking the magnetization of the small particles versus the whole magnetization as an adequate mixing parameter. With the magnetization $m_{\text {binary }}\left(x_{s}\right)=\left[x_{s} \chi_{s}+\left(1-x_{s}\right) \chi_{b}\right] B$ this leads to the formula for $\hat{x}_{s}$

$$
\hat{x}_{s}=\frac{m_{s}}{m_{\text {binary }}}=\frac{x_{s} \delta_{\chi}}{1+x_{s}\left(\delta_{\chi}-1\right)} .
$$

Alternatively, it is possible to assume the existence of an effective hard-disk radius of the dipolar particles. The effective radius $r_{s}$ and $r_{b}$ would be different for the two sizes, so that the force $F_{\alpha}(r)=\frac{\partial u_{\alpha \alpha}}{\partial r}$ on a dipolar test-particle at the effective radius is the same: $F_{s}\left(r_{s}\right) \stackrel{!}{=} F_{b}\left(r_{b}\right)$. Thus an effective hard disk ratio can be defined.

$$
\delta_{\mathrm{R}, \mathrm{eff}}:=\frac{r_{s}}{r_{b}}=\sqrt{\delta_{\chi}}
$$

which leads to eqn (6) again when eqn (3) for hard disks is used.

To make it easy to compare the hard disk system with the dipolar system, the data with the same $\delta_{R}$ and $\delta_{R}$, eff are plotted with the same symbols.

\subsection{Perturbation of a one-component system}

For predominant concentrations of one component, one might assume that the other can be seen as a perturbation of a onecomponent system. For this only the diagonal entry of the majority component of the structure factors $S$ and $F$ has to be taken into account and the shear modulus can be calculated with the one-component formula:

$$
\frac{G_{\infty \alpha \alpha}}{n k_{B} T}=\frac{1}{n} \frac{1}{32 \pi} \int_{0}^{\infty} k^{3}\left(\frac{1}{S_{\alpha \alpha}(k)} \frac{d S_{\alpha \alpha}(k)}{d k} \frac{F_{\alpha \alpha}(k)}{S_{\alpha \alpha}(k)}\right)^{2} d k .
$$

By this, the minority-component is taken into account by the changes it makes in the structure and glass form factors of the majority-component.

\section{Calculational details}

Our calculations of the shear moduli build on previous MCT calculations of the density correlators done for both systems. ${ }^{15-18}$

For the hard disk system, ref. 15 used the Percus-Yevick approximation for the partial structure factors $S$ and calculated the nonergodicity parameter $F$, the localization lengths, and $\phi_{c}$ in mode coupling approximation ${ }^{15}$ for $x_{s} \in[0.01,0.99]$ and $\delta_{R} \in$ $[0.3,0.9]$.

In the dipolar system, Monte-Carlo techniques were used to obtain the static structure factors. ${ }^{18}$ The glass transition surface $\Gamma_{c}\left(\delta_{x}, x_{s}\right)$ and the nonergodicity parameters were calculated with MCT for $x_{s} \in[0.1,0.9]$ and $\delta_{\chi} \in[0.1,0.6]$.

Every input into our calculations consists of the entries of the $2 \times 2$ matrices $S(k)$ and $F(k)$. For the dipolar system, these consist of 250 discrete values for $k, k=0.0606,0.2606, \ldots, 49.8606$. For the hard disk system, they consist of 250 discrete values for $k=0.0909,0.3909, \ldots, 74.7909$. These form the entry to a program, which calculates the plateau shear modulus $G_{\infty} /\left(n k_{B} T\right)$ using eqn (1). For this $S$ is transformed into the normalized direct correlation function $C$ using the OrnsteinZernike relation. The derivative and the integration are done by spline interpolation with GSL (GNU Scientific Library).

\section{Results and discussion}

\subsection{Hard disks}

The critical surface $\phi_{c}\left(\delta_{R}, x_{s}\right)$ of the packing fraction (the values at the glass transition) has been presented before, ${ }^{15}$ but is shown 


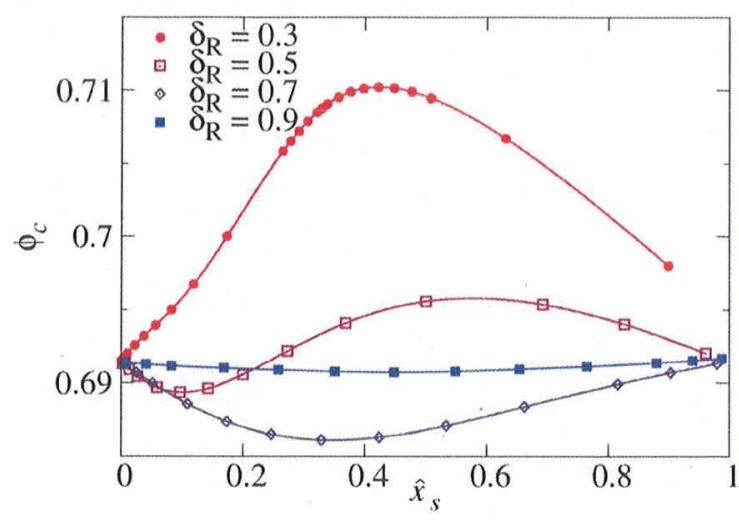

Fig. 1 Critical packing fraction $\phi_{c}$ for hard disks versus relative volume fraction of smaller disks. For small size differences (size ratio $\delta_{R}$ close to one) mixing stabilizes the glassy regime. For small $\delta_{R}$ the liquid is stabilized with a maximum at $\hat{x}_{s} \approx 0.4$.

in Fig. 1 for completeness. For small size disparities $\left(\delta_{R}>0.5\right), \phi_{c}$ is reduced by mixing. Thus the glass is stabilized. For small $\delta_{R}$ and high $\hat{x}_{s}$ the critical surface has a maximum. Here the liquid is stabilized. The critical surface is similar to the surface found for the three-dimensional system by Götze and Voigtmann although the liquid-stabilizing regime starts in three dimensions at a higher size ratio $\delta_{R}<0.7^{8}$.

The plateau shear modulus $G_{\infty} /\left(n k_{B} T\right)$ was calculated on the critical surface $\phi_{c}\left(x_{s}, \delta_{R}\right)$ using eqn (1) and can be seen in Fig. 2. The magnitude of the plateau modulus as well as its variation and form are about the same as in the three-dimensional system. ${ }^{8}$ Only the maximum is lower for the three-dimensional system, it increases only a bit above the limiting one-component value. Ideal mixing would lead to a horizontal line, as $G_{\infty}$ is plotted in units of $\left(n k_{B} T\right)$, and thus the change in particle number density is rescaled away. A general non-ideal mixing trend is the weakening of the elastic strength. We chose the term 'softening' here for the

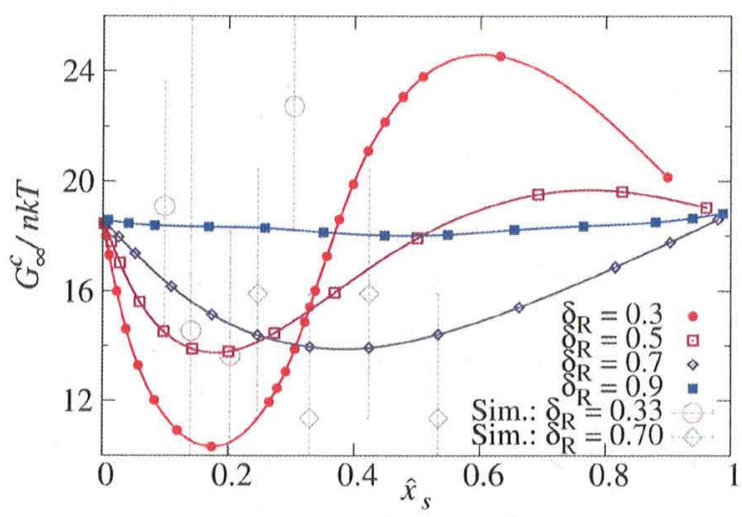

Fig. 2 Plateau shear modulus $G_{\infty}^{c}\left(x_{s}\right)$ for hard disks along the glass transition lines shown in Fig. 1. Softening, viz. a decrease of $G_{\infty}$ through mixing, is observed in the whole regime except for small size ratios $\delta_{R}$ and large relative volume fractions $\hat{x}_{s}$. Also, data from simulation for two size ratios $\left(\delta_{R}=5 / 7\right.$ at $\phi=0.79$, and $\delta_{R}=1 / 3$ at $\left.\phi=0.81\right)$ are included; to correct for the underestimation by theory, the simulation data are divided by a factor 2.2 (open symbols with error bars). lowering of the elastic constant $G_{\infty}$ through mixing, and distinguish it from 'plasticizing'. Plasticizing is connected to a change in the viscosity and structural relaxation time, and thus can be connected to the shift of the glass transition density or temperature, as shown in Fig. 1 and discussed in.,15

The shear modulus generally shows softening except in the region starting around $\delta_{R} \leq 0.5$ and $\hat{x}_{s}>0.5$. This weakening of the glass can be explained as a dilution of the majority-component, which continues to form the load bearing glassy structure. ${ }^{20}$ Fig. 3 shows the diagonal components of the structure and glass form factors which enter our results. ${ }^{15}$ For low $\hat{x}_{s}$, structure $S_{b b}(k)$ and form factor $F_{b b}(k)$ of the bigger disks change smoothly from the one-component results as if the density of the majoritycomponent was reduced. The length scales in $S_{b b}(k)$ and $F_{b b}(k)$ remain constant, the modulation in $S_{b b}(k)$ decreases, and $F_{b b}(k)$ increases and obtains more of the character of a diluted component. This also holds when small disks are diluted by (somewhat) larger ones, viz. for large $\hat{x}_{s}$ and not too-disparate disk sizes. For $\delta_{R}=0.7$ and $=0.9$ the same is true for a dilution of a majority-component of small disks: $S_{s s}(k)$ and $F_{s s}(k)$ show the just described variation, and the shear modulus decreases with lowering $\hat{x}_{s}$. The corresponding $S_{s s}(k)$ and $F_{s s}(k)$ are not displayed here, but will be shown for an equivalent situation from Monte Carlo simulations of the dipolar system in Fig. 10. Perturbative calculations based on eqn (7) recover the softening semi-quantitatively, reinforcing our interpretation of it as a dilution-effect of the majority-component.

For disparate sizes and a majority component of smaller disks, especially for $\delta_{R}=0.3$ and $\hat{x}_{s} \geq 0.4$ in Fig. 2 , the mixture becomes appreciably stiffer than its constituting species. This anomalous mixing effect may have two physical origins: (i) a structuring of the mixture on intermediate length scales, or (ii) the induction of an attractive interaction by one species among particles of the other. The following observations point to the former effect $(i)$ :

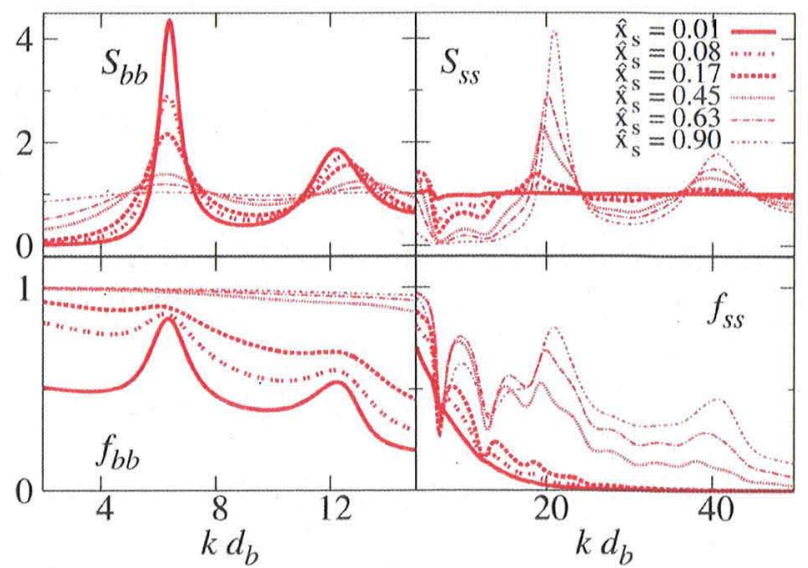

Fig. 3 Structure factors and nonergodicity parameters of hard disks for $\delta_{R}=0.3$ and for various volume ratios $\hat{x}_{s}$. For a dilution of the bigger disks, $\hat{x}_{s}<0.2$, the modulation of the majority structure factor $S_{b b}$ decreases while the corresponding nonergodicity parameter $f_{b b}=F_{b b} / S_{b b}$ approaches a bell-shape. For large $\hat{x}_{s}$, the small disk structure factor $S_{S s}$ and nonergodicity parameter $f_{s s}=F_{s s} / S_{s s}$ quickly obtain structure on length scales beyond the average small distance; $f_{s s}$ develops a pre-peak around $k d_{b}=7$. 
the structure factor $S_{s s}$ of the majority of small disks and especially the corresponding glass form factor $f_{s s}=F_{s s} / S_{s s}$ obtain $k$ dependent structure on the distance scale connected to the average separation of big disks. In Fig. 3 for $\delta_{R}=0.3$, the main peak in the partial structure factor of large disks remains at a constant $k d_{b} \approx 7$, the main peak in $S_{s s}$ lies around $k d_{b}=k d_{s} / \delta_{R}$ $\approx 21$ and the form factor $f_{b b}=F_{b b} / S_{b b}$ changes little above $\hat{x}_{s} \geq$ 0.5 . However, the nonergodicity parameter of small disks attains a prepeak around $k d_{b} \approx 7$ upon adding only small volume amounts of larger disks, viz. lowering $\hat{x}_{s}$ somewhat below unity. The increase of this pre-peak upon lowering $\hat{x}_{s}$ apparently drives the increase of $G_{\infty}$. While the $k^{d+1}$-prefactor in the MCT integral (1) weighs large wavevector contributions more strongly, the prepeak in $f_{s s}(k)$ is coupled to the structure functions of the large species. A possible explanation is that the majority of small disks arrests into clusters whose size is given by the minority of larger disks. Together the clusters and larger disks form a high density glass (note that the total packing fraction for the glass transition at these parameters is quite high in Fig. 1), which exhibits a larger modulus. This explanation based on the density structure functions is supported by snapshots of simulations (see Fig.6) and points to a true anomalous mixing effect causing the glass stiffening.

For three-dimensional hard sphere systems, an increase of the shear modulus for small $\delta_{R}$ and large $\hat{x}_{s}$ could be explained with a different effect (ii), namely a glass-glass transition caused by a depletion attraction induced among the bigger disks by the smaller ones. ${ }^{21}$ The depletion attraction characteristically increases the modulus strongly and leads to different glass types. $^{21,22}$ This leads to the question whether there are signs for a glass-glass transition near the maxima in the two-dimensional system.

An indicator for glass-glass transitions within MCT is that the exponent parameter $\lambda$ has values close to one. ${ }^{7,21}$ A plot of that variable (Fig. 4) shows that there is a maximum for small $\delta_{R}$ in the region where the shear modulus changes from lower than the boundary value to higher. But the maximum of $\lambda_{c}$ at 0.87 is not conclusive. Another variable that might change at a glass-glass transition is the localization length of the small and big particles $r_{s, s}$ and $r_{s, b}$ in Fig. $4 .^{7,21}$ The boundary values of the localization length agree (within a small error margin) with the ones of the mono-component system as can be expected..$^{19}$ Otherwise they show nothing special. So, while $\lambda$ and $G_{\infty}$ might indicate a closeby glass-glass transition, the glass hardening, viz. the increase of the elastic constant of a small-component glass upon addition of much bigger disks, is present for not too disparate size ratios $\delta_{R}$ $\leq 0.5$, already, and thus in our opinion presents a true mixing effect not dependent on the possibility of additional glass-glass transitions.

In order to assess the density dependence of $G_{\infty}$ when crossing a glass transition and moving deeper into the glass, we studied the square-root dependence on the separation parameter eqn (2). While it provides only an asymptotic description, the magnitude of $H_{G}$ indicates where strong density dependences can be expected. The critical amplitude $H_{G}$ of the shear modulus in Fig. 5 parallels the shape of $G_{\infty}^{c}$ along the glass transition lines. Where $G_{\infty}^{c}$ is large, also a stronger density dependence is predicted in the glass. This shows that the trend for a maximum and its slope are increased further in the glass.

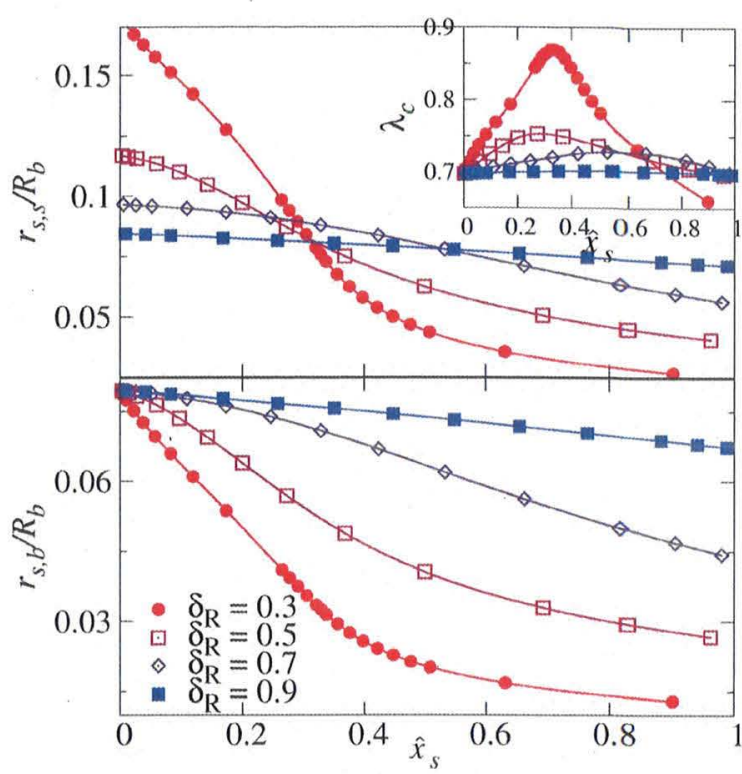

Fig. 4 Localization length $r_{s}$, $\alpha$ of small ( $\alpha=s$, top) and big ( $\alpha=b$, bottom) particles for hard disks along the glass transition lines from Fig. 1. The negative slope becomes steeper for decreasing $\delta_{R}$. Small panel: $\lambda$ for hard disk mixtures. The maximum at $\hat{x}_{s} \approx 0.3$ could stem from a glass-glass transition at $\delta_{R}<0.3$.

Studying mixing effects on glass properties, the question of possible nucleation of crystallites needs to be addressed. Small crystallites obviously would strongly contribute to the viscoelastic behavior. Fig. 6 shows snapshots from Brownian dynamics simulations of the hard disk mixtures. ${ }^{17}$ Because of crystallization, observed by checking the structure factors for qualitative deviations from the Percus-Yevick approximation, only a smaller parameter region excluding the monodisperse limits was accessible. The presented systems were judged free of crystallites. It is noteworthy, that for the two size ratios, $\delta_{R}=1 / 3$ and $5 / 7 \approx 0.7$, only the region where the larger majoritycomponent is diluted could be supercooled, while mixtures with more small disks crystallized too quickly in the simulations. Data on the elastic shear moduli of these simulated mixtures were

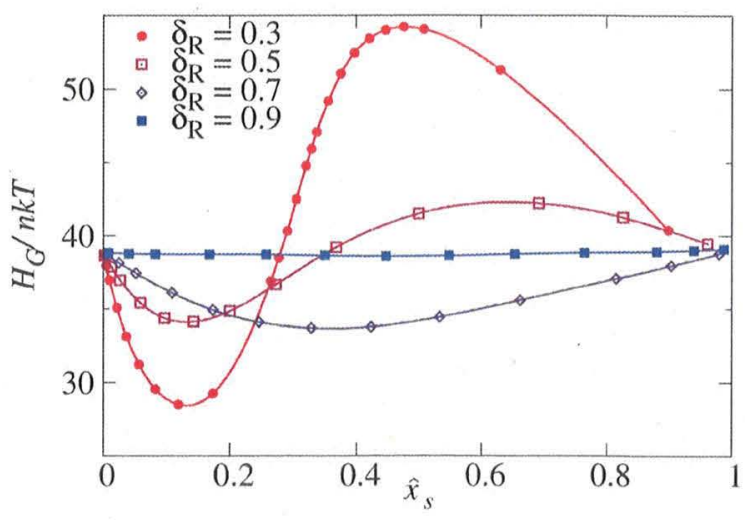

Fig. 5 Critical amplitude $H_{G}$ of $G_{\infty}\left(x_{s}\right)$ for hard disks. The effects of $G_{\infty}$ increase deeper in the glassy regime. 

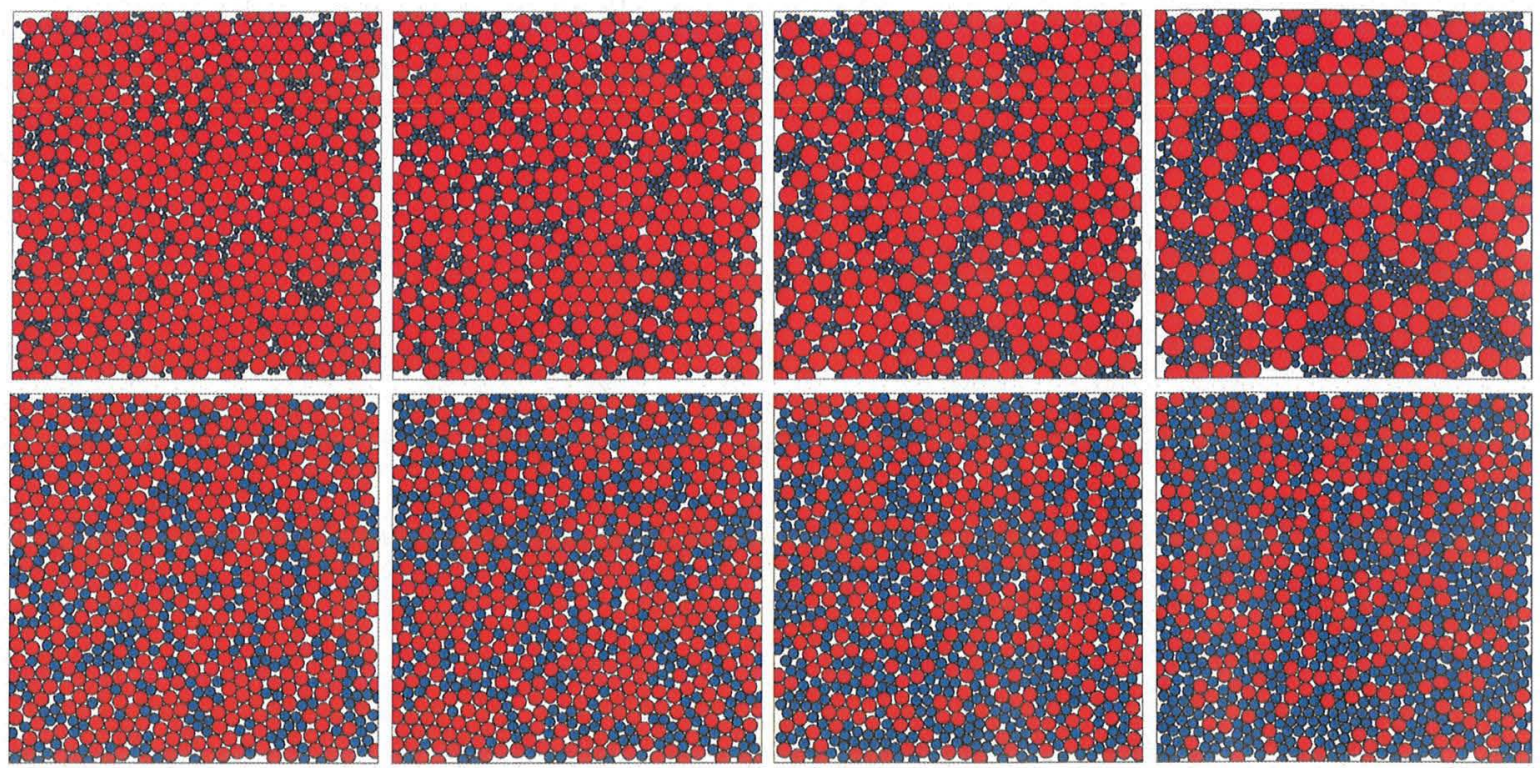

Fig. 6 Some configurations for hard disks with (top, left to right) $\delta_{R}=1 / 3, \hat{x}_{s}=0.10,0.14,0.20,0.30$ and (bottom) $\delta_{R}=5 / 7 \approx 0.7$, $\hat{x}_{s}=0.23,0.33,0.42,0.53$, where crystallization could be neglected during the Brownian dynamics simulations. ${ }^{17}$ For the disparate disks at the highest concentration of small ones, clusters of small disks are formed which are of comparable size to the larger disks.

obtained from the $\alpha$-relaxation strength in $G(t)$ in a fluid state close to the glass transition, and thus carry rather large error bars. Simulations were done at $\phi=0.79$ for $\delta_{R}=5 / 7$ and $\phi=0.81$ for $\delta_{R}=1 / 3 .{ }^{17}$ The data are included in Fig. 2. The critical packing fractions $\phi_{c}$ are not known for most of the mixtures, ${ }^{17}$ but it is known that MCT overestimates the trend to vitrification. ${ }^{7}$ Considering the moduli, we find that the first principles MCT calculation without adjustable parameters underestimates the elastic shear modulus by around a factor of two. The qualitative trends, however, are correctly predicted by MCT. The overall relative variation is close. The mixture of more similar disks shows less change of the shear modulus with mixing parameter $\hat{x}_{s}$, while the mixture of more disparate sizes exhibits a more rapid variation. For small amounts of small disks, its modulus is lower than the one of the more similar mixture, while upon adding small disks it rises above it.

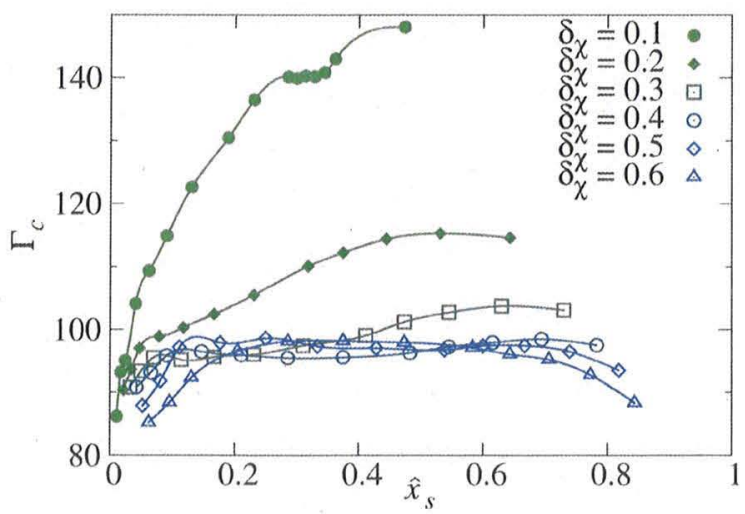

Fig. 7 Critical parameter $\Gamma_{c}$ for the dipolar system. Mixing stabilizes the fluid throughout. $\Gamma \mathrm{c}\left(\hat{x}_{s}, \delta \chi\right)$ shows a maximum for $\hat{x}_{s} \approx 0.5$ and $\delta_{x}=0.1$.

\subsection{Dipolar system}

Turning to the dipolar system we first recall its glass transition lines for completeness. They were obtained using structure factor input from Monte Carlo simulations. The critical parameter $\Gamma_{c}$ of the dipolar system shows a stabilization of the fluid regime for all parameters studied. ${ }^{18}$ This is especially pronounced for $\delta_{\chi}=0.1$ and $\hat{x}_{s} \approx 0.5$, where the critical parameter has a maximum. In Fig. 7 the glass transition surface is given, but plotted in $\hat{x}_{s}$ instead of $x_{s}$. A comparison with a plot in $x_{s}{ }^{18}$ shows that this renders the diagram more symmetric, especially for $\delta_{\chi}=$ 0.1 . At the boundaries, the critical parameter should be the same again, so the $\Gamma_{c}$ is supposed to drop again after the maximum. In

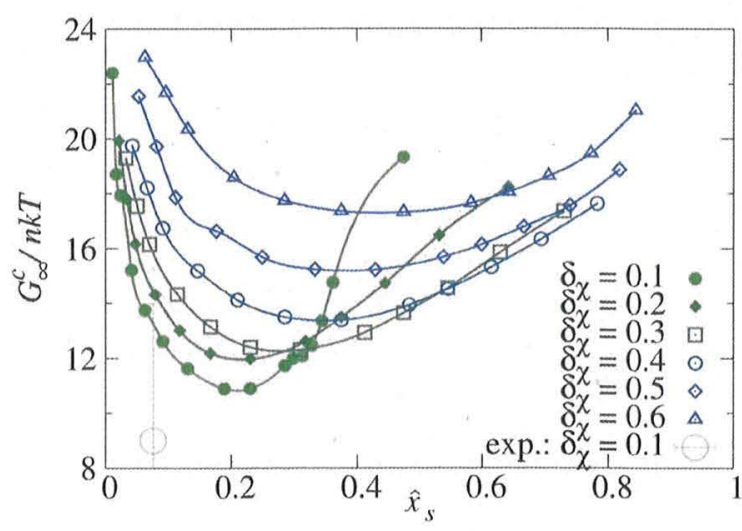

Fig. 8 Glass shear modulus $G_{\infty}^{c}\left(x_{s}\right)$ for the dipolar system along the glass transition lines shown in Fig. 7. Mixing softens the glass with increasing effect the smaller the size ratio $\delta_{\chi}$ is for small $\hat{x}_{s}$. An experimentally measured data point is included with error bar arising from the uncertainty in $\Gamma_{c \cdot}{ }^{13}$ 
Fig. 8 the plateau shear modulus of the dipolar system is shown. The glassy regime is not only suppressed by mixing, the glass also softens throughout by mixing. As we study $G_{\infty}^{c}$ along the glass transition line, this implies that the elastic constant is softened by mixing even though the interaction parameter $\Gamma_{c}$ is higher when the mixture vitrifies. This statement holds for the whole parameter regime we could access by Monte Carlo simulations, which however is restricted by crystallization. For small $\delta_{\chi}$ the curve has a similar behavior but the minimum is lower and shifts to lower $\hat{x}_{s}$. It is not clear whether the shear modulus might increase to a maximum before reaching the monodisperse limiting value, since simulation could only be done up to $x_{s}=0.9$, corresponding to $\hat{x}_{s} \approx 0.5$ for $\delta_{x}=0.1$.

The critical amplitudes of the shear modulus for different mixtures shown in Fig. 9 again imply that the mixtures with higher modulus at the transition $\left(G_{\infty}^{c}\right)$ exhibit a stronger dependence on $\Gamma$ when moving into the glass at fixed interaction ratio. $H_{G}$ varies roughly in parallel with $G_{\infty}^{c}$ along the transition lines.

As for the hard-disk system, a perturbation calculation using eqn (7) shows that the structural and elastic change is visible in the majority components except for the incremental strengthening of $G_{\infty}$ for very disparate species. Fig. 10 shows the partial structure and glass form factors for concentrations $\hat{x}_{s}$, where the majority component can be studied. In either case, regardless whether the smaller (viz. less interacting particles) or bigger ( $v i z$. larger dipole moment) particles are in majority, mixing-in more of the minority component smoothens out the majority partial structure factor, increases the majority glass form factor towards the bell shape of an incoherent nonergodicity parameter, and otherwise causes little shift in the relevant length/wave vector scales. Like explained for the hard disks, and there shown for the case of a majority component of larger disks only (see Fig. 3), this effect weakens the elasticity stored in the majority component which dominates the total elastic constant. For dipolar particles of similar interaction strength, a minimum in $G_{\infty}$ results which lies around $\hat{x}_{s}=1 / 2$, while it shifts to the side of the more strongly interacting dipoles for more disparate $\delta_{\chi}$. Different to the case of a three-dimensional colloid-polymer mixture, where the depletion-attraction increases the non-ergodicity parameters of the colloidal (majority) component also, in the present case the elastic constant is softened for all mixtures studied. In

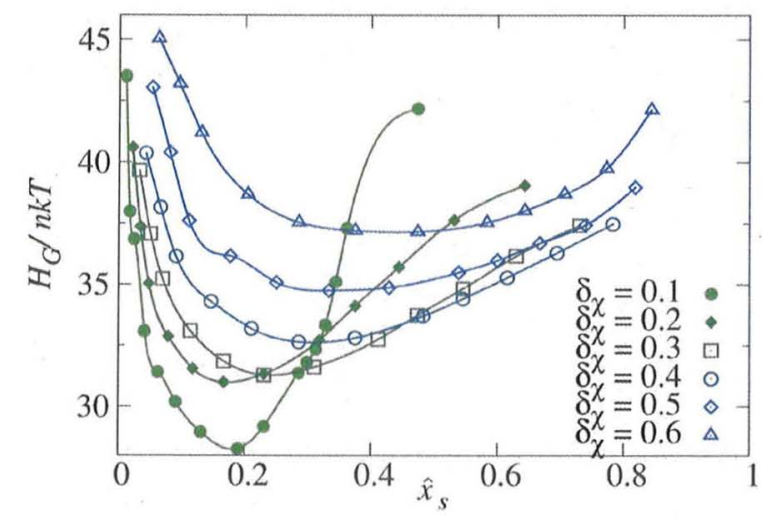

Fig. 9 Critical amplitude $H_{G}$ of $\mathrm{G}_{\infty}\left(\hat{x}_{s}\right)$ for the dipolar system shows that the mixing effects of $G_{\infty}$ are enhanced deeper in the glass.

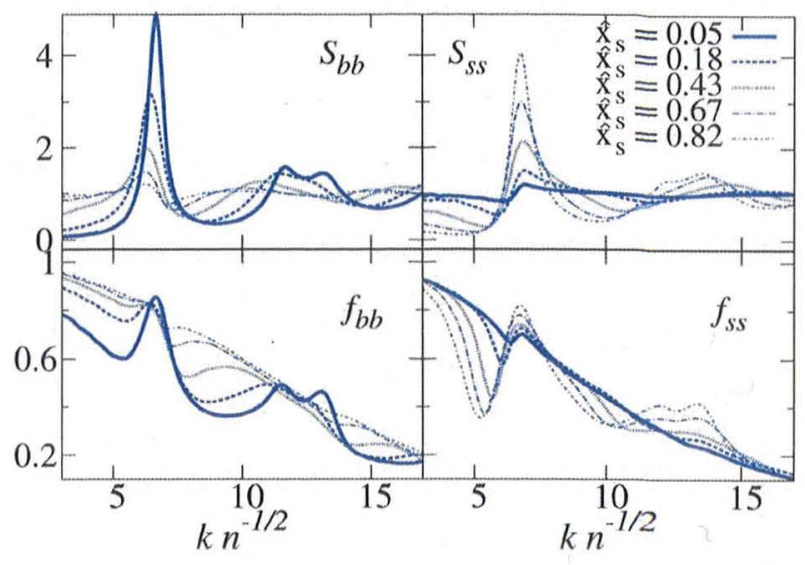

Fig. 10 Structure factors and nonergodicity parameters of the dipolar system for $\delta_{\chi_{.}}=0.5$ and various interaction ratios: For a dilution of one component, the maxima of the majority $S_{\alpha \alpha}$ decrease while the corresponding majority form factor $f_{\alpha \alpha}=F_{\alpha \alpha \alpha} / S_{\alpha x}$ approaches the form of an incoherent $f$. This explains the softening upon mixing in a 'big' or 'small' component.

both systems, the localization length shrinks upon mixing, see Fig. 11.

As in the hard-disk system, a glass-glass transition could be a possible explanation for the differing behavior of the shear modulus for small $\delta_{x}$. Testing the quantities where such a scenario was revealed in colloid-polymer mixtures, the present simulation and theoretical data remain inconclusive, however. The localization length (Fig. 11) of the big particles $r_{s, b}$ shows a maximum for small $\hat{x}_{s}$ that cannot be seen in the hard disk

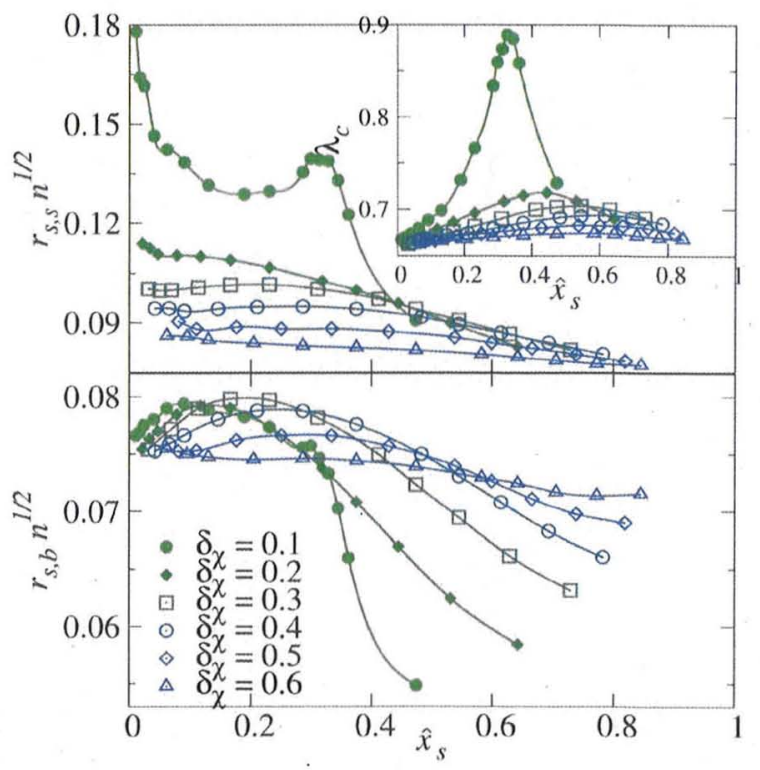

Fig. 11 Localization length $r_{s},{ }_{\alpha}$ of small $(\alpha=s$, top) and big $(\alpha=b$, bottom) particles for the dipolar system. The curve for $\delta_{x}=0.1$ shows a maximum for $\hat{x}_{s}=0.3$. Small panel: $\lambda_{c}$ for the dipolar system. The increase of the maxima for fixed $\delta_{x}$ seem to indicate a glass-glass transition at $x_{s} \approx 0.8\left(\hat{x}_{s}=0.33\right)$ and $\delta_{\chi}<0.1$. 

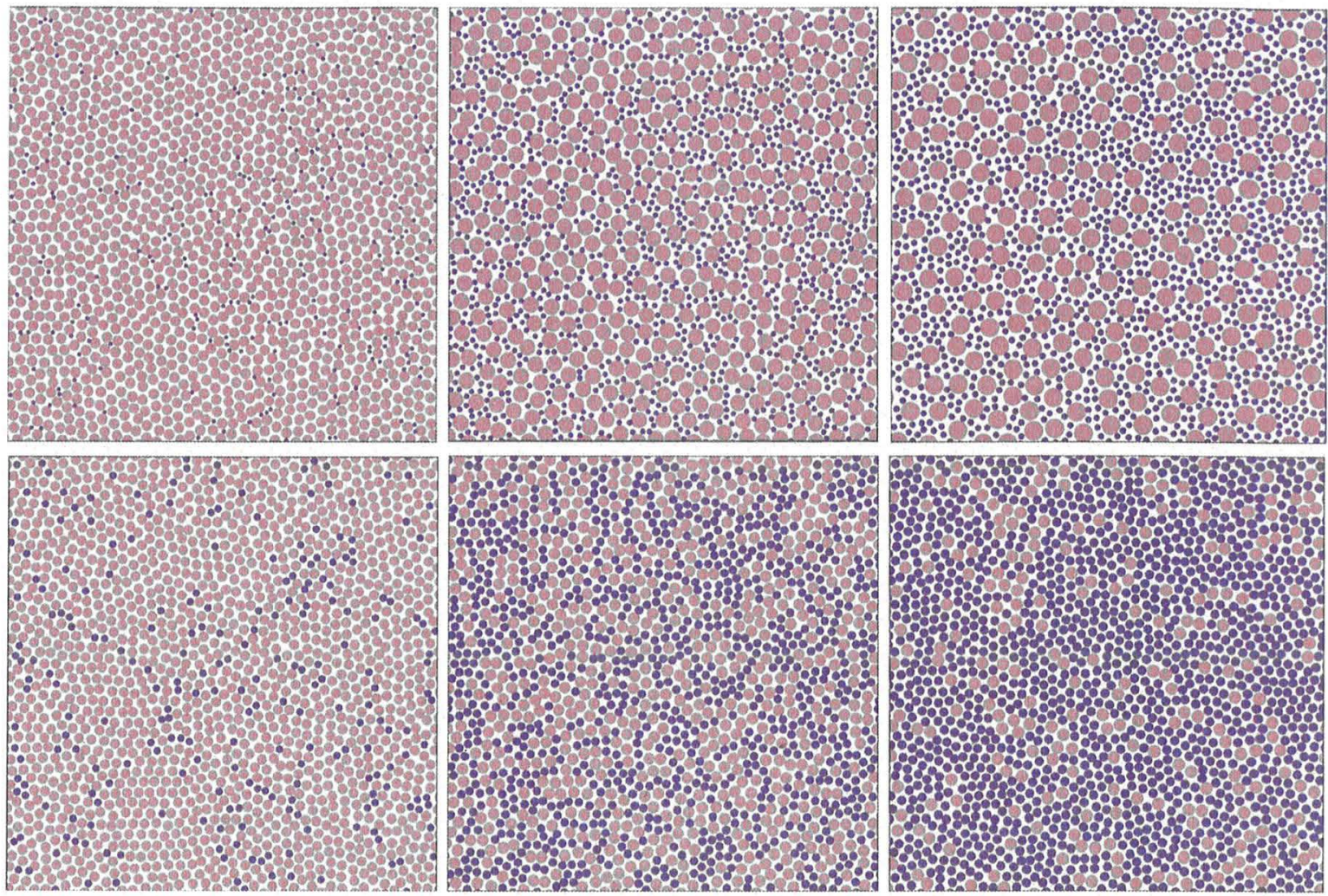

Fig. 12 Some configurations for dipolar particles with (top, left to right) $\delta_{x}=0.1, \hat{x}_{s}=0.02,0.19,0.36$ and (bottom) $\delta_{x}=0.5, \hat{x}_{s}=0.08,0.43,0.74$. For either $\delta$, this corresponds to one point at the smallest $\hat{x}_{s}$, one in the minimum of $G_{\infty}$ and one with bigger $\hat{x}_{s}$. The ratio of radii is chosen as $\delta_{\mathrm{R}, \text { efr }}=\sqrt{\delta_{\chi}}$ and the absolute value is chosen so that the packing fraction equals that of the corresponding hard disk system at the glass transition.

system. The localization length of the small particles shows a maximum for $\delta_{x}=0.1$ and $\hat{x}_{s} \approx 0.3$. $\lambda_{c}$ in Fig. 11 shows a more pronounced maximum for small $\delta_{\alpha}$ than the hard-disk system. So both the localization length, $G_{\infty}$ and $\lambda$ show some behavior that might indicate a glass-glass transition. Future work is required to test for the fascinating possibility of polyamorphism in this system, which offers the unique possibility for microscopic experimental investigation. ${ }^{12,13}$

In Fig. 12, a plot of particle configurations taken from the Monte Carlo simulations is shown, to see if the configuration for small $\delta_{\chi}$ and large $\hat{x}_{s}$ differs from others. This would also be an indicator for a glass-glass transition. Disk sizes are chosen according to the size ratio $\delta_{\mathrm{R} \text {, eff }}$, deduced from the interparticle forces in Sect. 2.4 and the absolute value is chosen so that the packing fraction equals that of the corresponding hard disk system at the glass transition.

The figure shows that for intermediate $\hat{x}_{s}$ the system has a randomly-mixed configuration. For large $\delta_{x}$ there is some small-scale crystallizing visible. Here we can see an effect found by Hoffmann et al:.23 The small particles form a sponge-like topology. The larger ones form (crystallizing) clusters. For small $\delta_{x}$ and large $x_{s}$ however, the big particles are quite homogeneously distributed with the smaller ones around them.

4.2.1 Comparison to experiment. Recently there have been measurements with the experimental system ${ }^{12}$ to determine the glass transition by looking at the change in the plateau shear modulus when $\Gamma$ is increased. According to MCT, this should jump from 0 to $G_{\infty} \neq 0$ at the glass transition.

From the experimental values ${ }^{13}$ with $\delta_{x}=0.1$ and $x_{s}=0.45$ (that is $\hat{x}_{s}=0.076$ ), the critical parameter $\Gamma_{c}$ can be estimated as 220 or 330 , depending on the interpretation which of two steps in the shear modulus is the glass transition. The experimental data is not directly measured but calculated using the equipartition theorem. The resulting formula for the plateau shear modulus is

$$
\frac{G_{\infty}}{n k_{B} T}=\lim _{\vec{q} \rightarrow 0}\left[q^{2}\left\langle\left|u_{\perp}(\vec{q})\right|^{2}\right\rangle\right]^{-1} .
$$

It is $G_{\infty}(\Gamma=220) /\left(n k_{B} T\right) \approx 5$ and $G_{\infty}(\Gamma=330) /\left(n k_{B} T\right) \approx 13$.

The numerical values calculated with $\mathrm{MCT}$ are $\Gamma_{c} \approx 110$ and $G_{\infty} /\left(n k_{B} T\right) \approx 13$ (see Fig. 7 and 8 ). While the shear modulus is almost the same, the critical parameter is only one half of the experimental value. But differences in the critical parameter of about a half of its value are known for MCT for other systems. ${ }^{7}$

\section{Conclusion}

In this publication the plateau shear moduli of two different twodimensional binary systems (a hard disk system and a dipolar system) were discussed at cuts through the glass transition surface. We summarize major similarities and differences observed.

We introduced a volume-ratio $\hat{x}_{s}$ of the dipolar particles that can be defined via an effective hard-disk radius for the particles 
or by the ratio of the magnetization of the particles. This leads to almost no overlaps in configurations where the particles are plotted as disks when the same packing fraction as for the glass transition of hard disks is used. Plotting the figures of the hard disk system and the dipolar system in volume-ratio symmetricizes the figures and make the plots of the two systems look very similar. This motivates our choice of $\hat{x}_{s}$ as mixing parameter instead of the number ratio $x_{s}$. However, there remain some differences between both systems. While the critical parameter $\Gamma_{\text {c }}$ of the dipolar system only increases above the one-component value, the critical packing fraction of the hard disks can also be decreased through mixing if the radii are similar. In the elastic constants, both systems show a softening effect (lessening of the shear modulus through mixing) that can be explained as a dilution effect. For small $\delta_{R}$ and large $\hat{x}_{s}$, however, the plateau shear modulus of the hard disk system develops a maximum, while the dipolar system shows a plateau-like region there.

Both systems show two regions with differing behavior.

Softening (lowering of the plateau shear modulus through mixing) is the dominant mechanism in the mixtures for not too disparate particles. It can be seen for both systems in region 1 . The softening becomes more pronounced, the bigger the differences in size are. The critical amplitude shows the same behavior, so further in the glass this effect is enhanced. The effect can be explained as a dilution of a one-component system, as is visible in the structure and glass form factors: Close to the boundary, $S$ and $F / S$ of the majority component have the same form as for the one-component system, only with a smaller amplitude.

In region 2 (with small $\delta_{\chi}$ and high $x_{s}$ ) the configuration of the particles in the dipolar system shows a change: here the big particles are distributed evenly with the smaller ones around them. In the hard disk system, clustering of the smaller disks becomes prominent. The plateau shear modulus increases above the one-component value for the hard disk system, and possibly also for the dipolar system. $H_{G}$ shows that this is increased deeper in the glass. The perturbation calculation misses or strongly underestimates the effect. So the explanation for the maximum is not a kind of dilution effect of the small particles but a change in structure. For the dipolar system it might be speculated whether the small particles, by their positions around the big ones, increase the effective radius of the big ones. For the hard disk system this has been suggested before as a kind of depletion effect, ${ }^{8}$ but it may also arise from the clustering of the smaller disks into rigid arrangements distributed among the larger disks. This may push the mixture deeper into the glass phase of a composite structure, resulting in a higher $G_{\infty}$.

The different behavior of many variables in the two regions leads to the question, whether there might be a glass-glass transition at $\delta_{\chi}<0.1$ or $\delta_{R}<0.3, \hat{x}_{s} \approx 0.3$, as has been suggested for the three-dimensional hard sphere system. ${ }^{21}$ The maxima of $\lambda$ in the boundary between the regions supports that (dipolar: $\lambda=0.96$ for $\delta_{\chi}=0.09, \hat{x}_{s}=0.29$ ). However, in the same region for the dipolar system the MCT algorithm showed a very slow convergence and might thus underestimate $\Gamma_{c}$ and so no conclusive answer can be given.

The comparison of the theoretical shear moduli with data taken from hard disk simulations and one experimental measurement is promising. Theory, without adjustable parameters, appears to underestimate the moduli at the glass transition by around $50 \%$, but appreciable difficulties still exist in determining $G_{\infty}$ in simulations. Qualitative trends agree broadly. The almost precise agreement with the experimental system appears fortuitous, as recent tests of the theory in three dimensions indicated errors of around $40 \% .{ }^{24}$

\section{Acknowledgements}

We thank T. Voigtmann and P. Keim for helpful discussions and comments on the manuscript. The work was supported in part by the Deutsche Forschungsgemeinschaft via FOR 1394.

\section{References}

1 A. Bunde, M. D. Ingram and P. Maass, J. Non-Cryst. Solids, 1994, 172, 1222.

2 F. Faupel, W. Frank, M.-P. Macht, H. Mehrer, V. Naundorf, K. Rätzke, H. R. Schober, S. K. Sharma and H. Teichler, Rev. Mod. Phys, 2003, 75, 237-280.

3 N. Willenbacher, J. S. Vesaratchanon, O. Thorwarth and E. Bartsch, Soft Matter, 2011, 7, 5777-5788.

4 C. A. Schuh, T. C. Hufnagel and U. Ramamurty, Acta Mater., 2007, 55, 4067-4109.

5 W. H. Wang, Adv. Mater., 2009, 21, 4524

6 H. W. Sheng, H. Z. Liu, Y. Q. Cheng, J. Wen, P. L. Lee, W. K. Luo, S. D. Shastri and E. Ma, Nat. Mater., 2007, 6, 192.

7 W. Götze, Complex Dynamics of Glass-Forming Liquids, A ModeCoupling Theory, Oxford University Press, 2009.

8 W. Götze and T. Voigtmann, Phys. Rev. E, 2003, 67, 021502.

9 T. Voigtmann, A. Meyer, D. Holland-Moritz, S. Stüber, T. Hansen and T. Unruh, Europhys. Lett., 2008, 82, 66001.

10 S. M. Chathoth, B. Damaschke, M. M. Koza and K. Samwer, Phys Rev. Lett., 2008, 101, 037801.

11 A. M. Puertas, C. D. Michele, F. Sciortino, P. Tartaglia and E. Zaccarelli, 2007, 127, p. 144906.

12 H. König, R. Hund, K. Zahn and G. Maret, Eur. Phys. J. E, 2005, 18, 287-293.

13 C. L. Klix, F. Ebert, F. Weysser, M. Fuchs, G. Maret and P. Keim, Phys. Rev., 2011, ArXiv:1108.2636, submitted.

14 G. Nägele and J. Bergenholtz, J. Chem. Phys., 1998, 108, 9893-9904.

15 D. Hajnal, J. M. Brader and R. Schilling, Phys. Rev. E, 2009, 80, 021503.

16 D. Hajnal, $\mathrm{PhD}$ thesis, Johannes Gutenberg-Universität Mainz, 2010

17 F. Weysser and D. Hajnal, Phys. Rev. E, 2011, 83, 041503.

18 D. Hajnal, M. Oettel and R. Schilling, J. Non-Cryst. Solids, 2011, 357 302-310.

19 M. Bayer, J. M. Brader, F. Ebert, M. Fuchs, E. Lange, G. Maret, R. Schilling, M. Sperl and J. P. Wittmer, Phys. Rev. E, 2007, 76, 011508.

$20 \mathrm{~T}$. Voigtman, 2011, private communication.

21 T. Voigtmann, Europhys. Lett., 2011, 96, 36006.

22 J. Bergenholtz and M. Fuchs, Phys. Rev. E, 1999, 59, 5706-5715.

23 N. Hoffmann, F. Ebert, C. N. Likos, H. Löwen and G. Maret, Phys. Rev. Lett., 2006, 97, 078301.

24 J. J. Crassous, M. Siebenbürger, M. Ballauf, M. Drechsler, D. Hajnal, O. Henrich and M. Fuchs, J. Chem. Phys., 2008, 128, 204902. 